\title{
General transient solution of the one-step master equation in one dimension
}

\author{
Stephen Smith* \\ School of Biological Sciences, University of Edinburgh, Edinburgh EH9 3JR, United Kingdom \\ Vahid Shahrezaei ${ }^{\dagger}$ \\ Department of Mathematics, Imperial College, London SW7 2AZ, United Kingdom \\ (Received 7 March 2015; published 16 June 2015)
}

\begin{abstract}
Exact analytical solutions of the master equation are limited to special cases and exact numerical methods are inefficient. Even the generic one-dimensional, one-step master equation has evaded exact solution, aside from the steady-state case. This type of master equation describes the dynamics of a continuous-time Markov process whose range consists of positive integers and whose transitions are allowed only between adjacent sites. The solution of any master equation can be written as the exponential of a (typically huge) matrix, which requires the calculation of the eigenvalues and eigenvectors of the matrix. Here we propose a linear algebraic method for simplifying this exponential for the general one-dimensional, one-step process. In particular, we prove that the calculation of the eigenvectors is actually not necessary for the computation of exponential, thereby we dramatically cut the time of this calculation. We apply our new methodology to examples from birth-death processes and biochemical networks. We show that the computational time is significantly reduced compared to existing methods.
\end{abstract}

DOI: 10.1103/PhysRevE.91.062119

PACS number(s): 02.50.-r, 82.20.-w

\section{INTRODUCTION}

The master equation (ME) describes the evolution of the probability distribution of states of a continuous-time Markov process and is commonly used to describe stochastic physical, chemical, or biological systems. ME could consist of countably infinite set of coupled differential equations, which makes it hard to solve analytically or numercially. There is renewed interest in solutions of the master equation as they can be used to describe stochastic dynamics of biochemical reaction networks inside living cells [1,2]. Indeed, it has been shown that the chemical master equation accurately describes the dynamics of well-stirred dilute chemical systems [3]. Full analytical solutions to master equations are rare. However, there are several approximate analytical methods, including the system size expansion [4], the Fokker-planck equation and corresponding Langevin equation [5-7], moment closure approximations [8-11], and time-scale separation [12,13].

Alternatively, statistically exact trajectories of the Markov process can be simulated using Gillespie's stochastic simulation algorithm (SSA) [14]. However, the SSA is not efficient in estimating probability distributions as it requires many simulations. As the ME is a linear equation, its solution can be written as the exponential of a transition matrix. Direct numerical solution of the ME requires calculation of the exponential of an infinite dimensional matrix. An error-controlled method based on projection of the ME to a finite state space is proposed that requires the numerical calculation of a finite dimensional matrix exponential [15]. The exponential of a matrix is calculated by the time-consuming process of obtaining the eigenvectors and eigenvalues and then computing the inverse of the eigenvector matrix [16,17]. A graph theoretic method has been recently proposed to

\footnotetext{
*s1436741@sms.ed.ac.uk

†v.shahrezaei@imperial.ac.uk
}

approximate the eigenvectors and eigenvalues of the transition matrix [18], which accelerates the calculation of matrix exponential but is applicable only to the reversible system $A+A \rightleftharpoons B$. A related method using Laplace transforms is proposed for the reversible reaction $A+B \rightleftharpoons C$ [19].

An approximate method that also uses Laplace transforms and builds on Ref. [20] has recently been derived and is applicable to all one-step, one-dimensional (1D) systems [21]. This method approximates the Laplace transform of the distribution using continued fractions and then approximates the inverse transform using the Trapezium rule. The authors also publish error bounds for their approximation of the true distribution. The method presented in this paper differs from the approach taken in Ref. [21] in the sense that it uses neither Laplace transforms nor continued fraction representation.

In this paper, we focus on solutions of the 1D, one-step ME. This type of ME has the form

$$
\dot{P}_{m}(t)=a_{m} P_{m-1}(t)+b_{m} P_{m+1}(t)+\left(-a_{m+1}-b_{m-1}\right) P_{m}(t),
$$

where $P_{m}(t)$ denotes the probability of having $m$ copies of the species of interest at time $t$, and $a_{m}, b_{m}$ are positive real-valued sequences. The generating function method has solved this ME when the sequences $a_{m}, b_{m}$ are constant or linear, for instance, if $a_{m}=m+3$, and $b_{m}=2 m$, which corresponds to all reactions having at most one reactant. However, in cases where the reactions have two or more reactants (or if complicated reaction rates such as Michaelis-Menten are used), the sequences are nonlinear and there is no general solution except for the steady state [4].

Here we show that the eigenvectors are not needed for the computation of the exponential of the transition matrix in this case, and only the eigenvalues must be computed, which we can achieve computationally if necessary [22]. We show in a computational plot that computational calculation of eigenvalues adds negligible time to the calculation. Thus we dramatically improve on brute force exponentiation. We 
illustrate the application of our novel technique with three biological examples and compare our results with exact simulations.

\section{MAIN RESULT}

A $1 \mathrm{D} M E$ is a countably infinite set of coupled ODEs of the form

$$
\dot{P}_{m}(t)=a_{m} P_{m-1}(t)+b_{m} P_{m+1}(t)+\left(-a_{m+1}-b_{m-1}\right) P_{m}(t) .
$$

Let $\tau$ be a large positive integer. Following the error-controlled method of Ref. [15], we truncate the ME at $P_{\tau-1}(t)$ and ignore all higher terms. We can then write $P_{m}(t)$ as a finite vector,

$$
P(t)=\left(P_{0}(t), P_{1}(t), \ldots, P_{\tau-1}(t)\right)^{T} .
$$

Then the ME can be written as

$$
\dot{P}(t)=A P(t)
$$

where for the transition matrix $A$ we have

$$
A=\left(\begin{array}{ccccc}
-a_{1} & b_{0} & 0 & 0 & \ldots \\
a_{1} & -a_{2}-b_{0} & b_{1} & 0 & \ldots \\
0 & a_{2} & -a_{3}-b_{1} & b_{2} & \ldots \\
0 & 0 & a_{3} & -a_{4}-b_{2} & \ldots \\
\vdots & \vdots & \vdots & \vdots & \ddots
\end{array}\right)
$$

The solution to this ODE is simply

$$
P(t)=e^{A t} P(0),
$$

but what we seek is a closed form expression for $P(t)$, and in particular, one which can be computed quickly and easily.

Computing the exponential of a matrix is an extremely difficult problem, in general we have to calculate the eigenvectors and eigenvalues of $A$. But the special tridiagonal form of the matrix $A$ allows us to bypass the eigenvector calculation. Cauchy's integral forumla for matrices [23] is, for any integrable function $f$ :

$$
f(A)=\frac{1}{2 \pi i} \oint_{\gamma} f(z)(z I-A)^{-1} d z,
$$

where $\gamma$ is any closed curve in $\mathbb{C}$, which contains the eigenvalues of $A$ in its interior. If we choose $f(z)=e^{z t} P(0)$, then we see that

$$
P(t)=\frac{1}{2 \pi i} \oint_{\gamma} e^{z t}(z I-A)^{-1} P(0) d z .
$$

Suppose now that the initial solution of the ME is $P_{m}(0)=Q_{m}$. Typically this distribution will be a Kronecker delta, $Q_{m}=$ $\delta_{m, m_{0}}$ for some $m_{0} \in \mathbb{N}$. We will assume this for now and later explain how to extend our results to more general initial distributions. We can simplify further with

$$
P_{m}(t)=\frac{1}{2 \pi i} \oint_{\gamma} e^{z t}(z I-A)_{m+1, m_{0}+1}^{-1} d z .
$$

A formula for $(z I-A)^{-1}$ exists, when $A$ is tridiagonal [24]:

$$
(z I-A)_{i, j}^{-1}=\left\{\begin{array}{ll}
b_{i-1} \ldots b_{j-2} \frac{p_{i-1}(z) q_{j+1}(z)}{p_{\tau}(z)} & i<j \\
\frac{p_{i-1}(z) q_{j+1}(z)}{p_{\tau}(z)} & i=j \\
a_{j} \ldots a_{i-1} \frac{p_{j-1}(z) q_{i+1}(z)}{p_{\tau}(z)} & i>j
\end{array},\right.
$$

where $p$ and $q$ are orthogonal polynomials defined recursively by

$$
\begin{aligned}
p_{i}(z) & =\left(z+a_{i}+b_{i-2}\right) p_{i-1}(z)-b_{i-2} a_{i-1} p_{i-2}(z), \\
p_{0}(z) & =1, \quad p_{1}(z)=z+a_{1}, \\
q_{i}(z) & =\left(z+a_{i}+b_{i-2}\right) q_{i+1}(z)-b_{i-1} a_{i} q_{i+2}(z), \\
q_{\tau+1}(z) & =1, \quad q_{\tau}(z)=z+b_{\tau-2} .
\end{aligned}
$$

Therefore,

$$
P_{m}(t)= \begin{cases}\frac{b_{m} \ldots b_{m_{0}-1}}{2 \pi i} \oint_{\gamma} e^{z t} \frac{p_{m}(z) q_{m_{0}+2}(z)}{p_{\tau}(z)} d z & m<m_{0} \\ \frac{1}{2 \pi i} \oint_{\gamma} e^{z t} \frac{p_{m}(z) q_{m_{0}+2}(z)}{p_{\tau}(z)} d z & m=m_{0} . \\ \frac{a_{m_{0}+1 \ldots a_{m}}}{2 \pi i} \oint_{\gamma} e^{z t} \frac{p_{m_{0}}(z) q_{m+2}(z)}{p_{\tau}(z)} d z & m>m_{0}\end{cases}
$$

We also have the following useful results about the eigenvalues of $A$. Assuming the system of interest is ergodic, then $A$ should be irreducible, so $a_{i}$ and $b_{i}$ should be nonzero for all $i$. As these parameters are also non-negative by definition, we have that $a_{i} b_{i-1}>0$ for all $i$, which permits the following result.

Let

$$
D=\left(\begin{array}{ccccc}
1 & 0 & 0 & 0 & \ldots \\
0 & \sqrt{\frac{b_{0}}{a_{1}}} & 0 & 0 & \ldots \\
0 & 0 & \sqrt{\frac{b_{0} b_{1}}{a_{1} a_{2}}} & 0 & \ldots \\
0 & 0 & 0 & \sqrt{\frac{b_{0} b_{1} b_{2}}{a_{1} a_{2} a_{3}}} & \ldots \\
\vdots & \vdots & \vdots & \vdots & \ddots
\end{array}\right)
$$

and let

$$
\bar{A}=\left(\begin{array}{ccccc}
-a_{1} & \sqrt{b_{0} a_{1}} & 0 & 0 & \ldots \\
\sqrt{b_{0} a_{1}} & -a_{2}-b_{0} & \sqrt{b_{1} a_{2}} & 0 & \ldots \\
0 & \sqrt{b_{1} a_{2}} & -a_{3}-b_{1} & \sqrt{b_{2} a_{3}} & \ldots \\
0 & 0 & \sqrt{b_{2} a_{3}} & -a_{4}-b_{2} & \ldots \\
\vdots & \vdots & \vdots & \vdots & \ddots
\end{array}\right) .
$$

Then,

$$
\bar{A}=D^{-1} A D .
$$

The matrix $\bar{A}$ is real and symmetric, and so it has real eigenvalues. Since $A$ and $\bar{A}$ are similar, they have the same eigenvalues, and so $A$ has real eigenvalues. Since the sub- and superdiagonals of A are nonzero, the matrix is irreducible and therefore has distinct eigenvalues.

Moreover, we know from the definition of the ME that the columns of $A$ each sum to zero. It follows that there is at least one row which is a linear combination of the others. Therefore $A$ has zero determinant, and so 0 is an eigenvalue of $A$.

We now show that the matrix $-\bar{A}$ is positive semidefinite. It follows from the Gershgorin Circle Theorem that if $a_{i+1}+b_{i-1} \geqslant-\sqrt{b_{i-1} a_{i}}-\sqrt{b_{i} a_{i+1}}$, then $-\bar{A}$ is positive semidefinite. But this is clearly true because the left-hand side is strictly positive while the right-hand side is strictly negative. So it follows that $-\bar{A}$ has exclusively non-negative eigenvalues, and so also $-A$ does. Then $A$ must have nonpositive eigenvalues. But zero is an eigenvalue, so the largest eigenvalue of $A$ must be zero.

With these results, we can now proceed further with the ME. By its definition, $p_{\tau}(z)$ is the characteristic polynomial of 
$A$, so we can write, for $\lambda_{i}$ eigenvalues of $A$,

$$
p_{\tau}(z)=\left(z-\lambda_{1}\right)\left(z-\lambda_{2}\right) \cdots\left(z-\lambda_{\tau}\right) .
$$

We can then use Cauchy's Residue Theorem:

$$
\oint_{\gamma} f(z) d z=2 \pi i \sum \operatorname{Res}\left(f, a_{k}\right)
$$

where $a_{k}$ are the singularities of $f$ inside $\gamma$. For us, these singularities are simply the eigenvalues $\lambda_{i}$, so

$$
\oint_{\gamma} e^{z t} \frac{p_{m}(z) q_{m_{0}+2}(z)}{p_{\tau}(z)} d z=2 \pi i \sum_{i=1}^{\tau} e^{\lambda_{i} t} \frac{p_{m}\left(\lambda_{i}\right) q_{m_{0}+2}\left(\lambda_{i}\right)}{\prod_{j \neq i}\left(\lambda_{i}-\lambda_{j}\right)} .
$$

So if we know the eigenvalues of $A$ then we can obtain $P_{m}(t)$ :

$$
P_{m}(t)= \begin{cases}b_{m} \ldots b_{m_{0}-1} \sum_{i=1}^{\tau} e^{\lambda_{i} t} \frac{p_{m}\left(\lambda_{i}\right) q_{m_{0}+2}\left(\lambda_{i}\right)}{\prod_{j \neq i}\left(\lambda_{i}-\lambda_{j}\right)} & m<m_{0} \\ \sum_{i=1}^{\tau} e^{\lambda_{i} t} \frac{p_{m}\left(\lambda_{i}\right) q_{m_{0}+2}\left(\lambda_{i}\right)}{\prod_{j \neq i}\left(\lambda_{i}-\lambda_{j}\right)} & m=m_{0} . \\ a_{m_{0}+1} \ldots a_{m} \sum_{i=1}^{\tau} e^{\lambda_{i} t} \frac{p_{m_{0}}\left(\lambda_{i}\right) q_{m+2}\left(\lambda_{i}\right)}{\prod_{j \neq i}\left(\lambda_{i}-\lambda_{j}\right)} & m>m_{0}\end{cases}
$$

For general, non-delta-type initial distributions $P_{m}(0)=Q_{m}$, the transient solution is given instead by $\tilde{P}_{m}(t)$, which satisfies

$$
\tilde{P}_{m}(t)=\sum_{m_{0}=0}^{\infty} P_{m}(t) Q_{m_{0}}
$$

We can obtain steady-state distributions by taking $t \rightarrow \infty$. We notice that the only term in the sum which does not depend on $t$ is the first, since $\lambda_{1}=0$, and all other terms tend to zero as $t$ grows. Since the system is ergodic, the value of $m_{0}$ which we take (or equivalently the function $Q_{m}$ ) is irrelevant, so we take $m_{0}$ large, allowing us to pay attention only to the $m<m_{0}$ expression, which is now

$$
P_{m}(t \rightarrow \infty)=b_{m} \ldots b_{m_{0}-1} \frac{p_{m}(0) q_{m_{0}+2}(0)}{\prod_{j \neq 1}\left(-\lambda_{j}\right)} .
$$

But the fraction $\frac{q_{m_{0}+2}(0)}{\prod_{j \neq 1}\left(-\lambda_{j}\right)}$ contributes the same to each term, so we ignore it as a normalizing factor. We can also do the same with product $b_{m} \ldots b_{m_{0}-1}$, which contributes the same as $\frac{1}{b_{0} \ldots b_{m-1}}$ up to normalization. Therefore we have a simplified expression:

$$
P_{m}(t \rightarrow \infty)=\frac{p_{m}(0)}{b_{0} \ldots b_{m-1}} .
$$

We can simplify this further, since one can prove by induction that $p_{m}(0)=a_{1} \ldots a_{m}$, which leads to the following simple expression:

$$
P_{m}(t \rightarrow \infty)=\frac{a_{m} \ldots a_{1}}{b_{m-1} \ldots b_{0}}
$$

up to normalization. The steady-state result is derived in Ref. [4] using a different method.

\section{EXAMPLES}

\section{A. Bacterial growth}

A model of bacterial growth with a source term can be used to model the bacterial population in a reservoir, or any situation

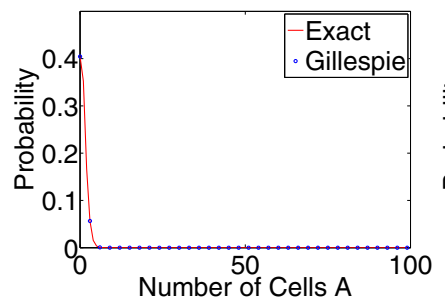

(a) $t=1$.

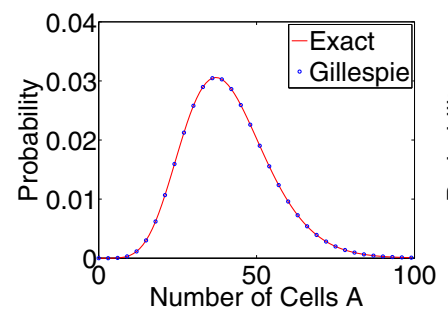

(c) $t=100$. (b) $t=10$.

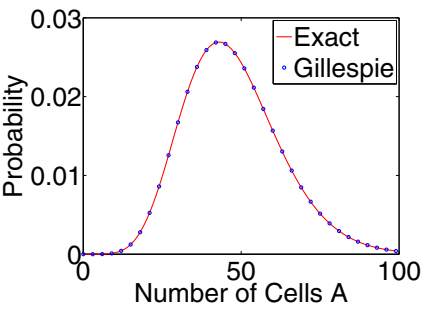

(d) $t=1000$.

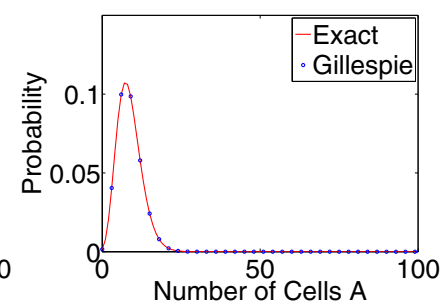

FIG. 1. (Color online) Probability distribution of $A$ in the bacterial growth system. Our method agrees excellently with the Gillespie algorithm as expected. Parameter values are $v=0.95, k=0.08, d=$ $0.1, A(0)=0$.

where bacteria are introduced at a constant rate:

$$
\emptyset \stackrel{v}{\underset{d}{\rightleftharpoons}} A, \quad A \stackrel{k}{\rightarrow} 2 A .
$$

The ME for this system is

$$
\begin{aligned}
\dot{P}_{m}= & v\left(P_{m-1}-P_{m}\right)+d\left[(m+1) P_{m+1}-m P_{m}\right] \\
& +k\left[(m-1) P_{m-1}-m P_{m}\right] .
\end{aligned}
$$

Therefore, in our notation,

$$
\begin{aligned}
& a_{m}=v+k(m-1), \\
& b_{m}=d(m+1) .
\end{aligned}
$$

Taking the limit $t \rightarrow \infty$ gives us that the number of $A$ molecules at steady state follows a negative binomial $\left(\frac{v}{k}, \frac{k}{d}\right)$ distribution, as observed in nature in Ref. [25]. In particular, this implies that

$$
P_{m}=\frac{\Gamma\left(m+\frac{v}{k}\right)}{m ! \Gamma\left(\frac{v}{k}\right)}\left(\frac{k}{d}\right)^{m}\left(1-\frac{k}{d}\right)^{\frac{v}{k}} .
$$

In Fig. 1 we compare our method with the numerical results from the Gillespie algorithm. Our method agrees perfectly with the Gillespie algorithm but can be computed in several orders of magnitude less time.

\section{B. Dimerization}

We consider an example of a closed dimerization reaction:

$$
A+A \underset{k_{2}}{\stackrel{k_{1}}{\rightleftharpoons}} B
$$

We have the conservation law $A+2 B=N$. This system has CME for species $A$ :

$$
\begin{aligned}
\dot{P}_{n}= & k_{1}\left[(n+2)(n+1) P_{n+2}-n(n-1) P_{n}\right] \\
& +\frac{k_{2}}{2}\left[(N-n+2) P_{n-2}-(N-n) P_{n}\right] .
\end{aligned}
$$




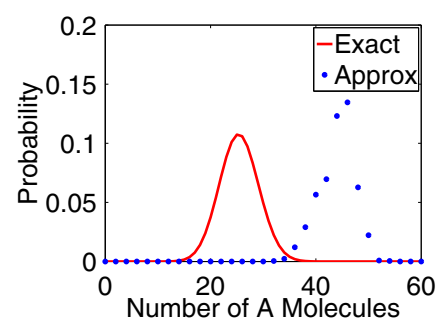

(a) $t=0.5$.

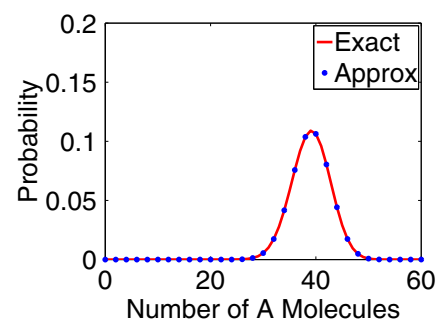

(c) $t=1$.

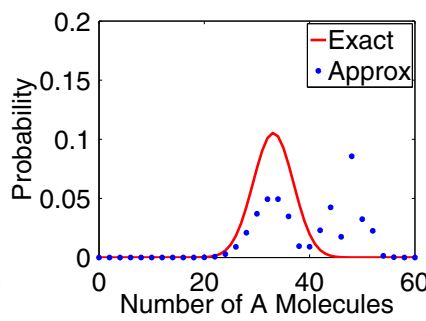

(b) $t=0.75$.

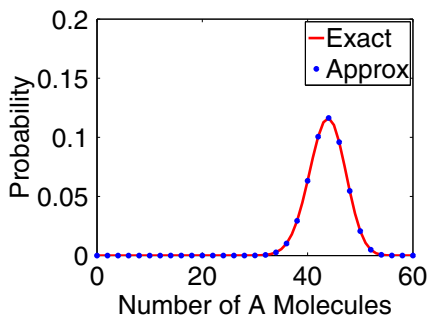

(d) $t=1.25$.
FIG. 2. (Color online) Probability distribution of $A$ in the dimerization system over time. Our method (red line) compared with using approximate eigenvalues (blue circles). Parameter values are $k_{1}=10^{-10}, k_{2}=2, N=120$.

In other words, in our notation:

$$
\begin{aligned}
& a_{n}=\frac{k_{2}}{2}(N-n+2) \\
& b_{n}=k_{1} 2(n+1)(2 n+1) .
\end{aligned}
$$

Figure 2 gives the time development of the probability distribution for this system, as computed using our exact method, compared with a faster version of our method using an approximation of the eigenvalues found in Ref. [18], namely

$$
\lambda_{n}=-k_{2} n-2 k_{1} n[2(N-2 n)+1], \quad n=0, \ldots, N .
$$

In general, the eigenvalues cannot be computed exactly, though they can usually be approximated.

While our method gives analytical expressions that need to be computed in practice, it has the advantage of being (to our knowledge) the fastest method yet of computing the probability distribution for one-species, one-step systems in time.

Figure 3 shows the computational time of calculating the solution to the dimerization example at the $3 \mathrm{sec}$ time point. When we are dealing with small reaction systems, such as when $\tau \approx 50$, we find that the direct matrix exponentiation takes around $10^{-2} \mathrm{sec}$, compared with $10^{-3} \mathrm{sec}$ for our method. In situations with larger molecule numbers, our method really starts to stand out. When we have $\tau \approx 5000$, direct matrix exponentiation typically takes around $5 \mathrm{~min}$, compared with $2 \mathrm{sec}$ for our method. We therefore have a dramatic improvement over the brute force method. In cases where the eigenvalues can be approximated, we have slightly better performance.

These results were computed using MATLAB on a computer with a $1.7 \mathrm{GHz}$ Intel i5 processor and $8 \mathrm{~GB}$ RAM. The matrix exponential was computed using MATLAB's expm function. When the eigenvalues needed to be approximated, the trideig function was used.

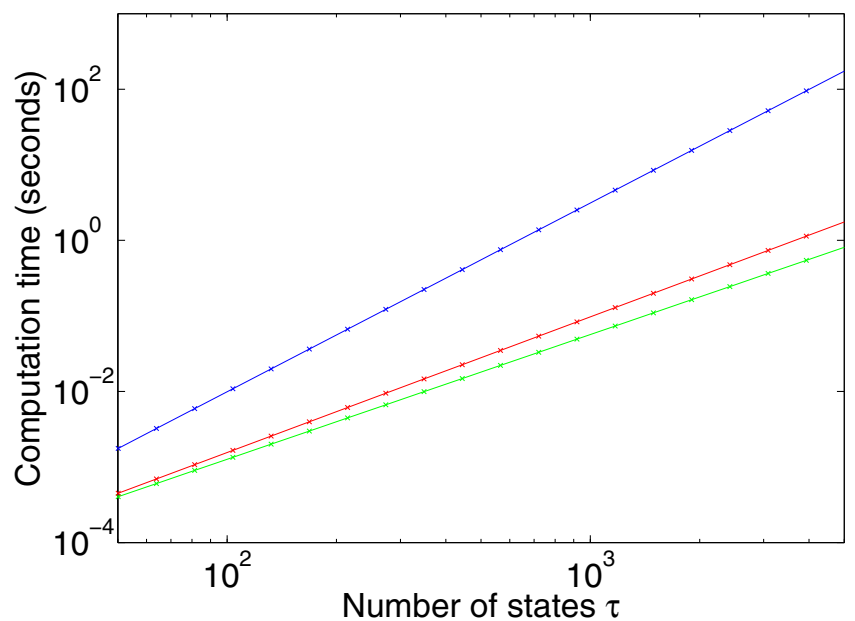

FIG. 3. (Color online) Log-log plot of Dimerization example computation time of our method (red line), our method using approximate solutions of eigenvalues (green line), and brute force matrix exponentiation (blue line), for a variety of system sizes. Leastsquares estimation suggests our method has an $O\left(\tau^{1.7}\right)$ computational complexity, while brute force exponentiation has an $O\left(\tau^{2.5}\right)$ complexity, compared with $O\left(\tau^{1.6}\right)$ for the approximation. Computational calculation of the eigenvalues adds negligible computation time to the process.

\section{Push-pull enzyme system}

A phosphorylation-dephosphorylation (or push-pull enzyme) system has been extensively studied as it can exhibit zero-order ultrasensitivity [26,27]:

$$
A+R \underset{k_{-}}{\stackrel{k_{+}}{\rightleftharpoons}} \tilde{A} \stackrel{v_{r}}{\rightarrow} A^{\star}, \quad A^{\star}+B \underset{k_{-}^{\prime}}{\stackrel{k_{+}^{\prime}}{\rightleftharpoons}} \tilde{A}^{\star} \stackrel{v_{b}}{\rightarrow} A .
$$

Here $A$ and $A^{\star}$ are the substrate and modified substrate respectively. $R$ and $B$ are modifying enzymes. We make the quasi-steady-state assumption (QSSA) that the concentration of the complexes $\tilde{A}$ and $\tilde{A}^{\star}$ does not change on the time scale in which the production of $A$ and $A^{\star}$ occurs. This will simplify the reaction system to the following [27]:

$$
A \underset{v_{b} B_{0} \frac{A^{\star}}{K_{M}^{\prime}+A^{\star}}}{\stackrel{v_{r} R_{0} \frac{A}{K_{M}+A}}{\rightleftharpoons}} A^{\star}
$$

where $K_{M}=\frac{v_{r}+k_{-}}{k_{+}}$, and $K_{M}^{\prime}=\frac{v_{b}+k_{-}^{\prime}}{k_{+}^{\prime}}$. Also, $R_{0}$ and $B_{0}$ denote total level of each enzyme respectively. Using the conservation law $A+A^{\star}=N$, the ME for $A$ is

$$
\begin{aligned}
\dot{P}_{m}= & v_{r} R_{0}\left(\frac{m+1}{K_{M}+m+1} P_{m+1}-\frac{m}{K_{m}+m} P_{m}\right) \\
& +v_{b} B_{0}\left[\frac{N-(m-1)}{K_{M}^{\prime}+N-(m-1)} P_{m-1}-\frac{N-m}{K_{M}^{\prime}+N-m} P_{m}\right] .
\end{aligned}
$$

This is effectively a 1D process, so in our notation

$$
a_{m}=v_{b} B_{0} \frac{N-m+1}{K_{M}^{\prime}+N-m+1}, \quad b_{m}=v_{r} R_{0} \frac{m+1}{K_{M}^{\prime}+m+1} .
$$

We can now use our method to very efficiently compute transient distributions of $A$ for a range of parameter values. Figure 4 shows how the mean value of $A$ approaches steady 


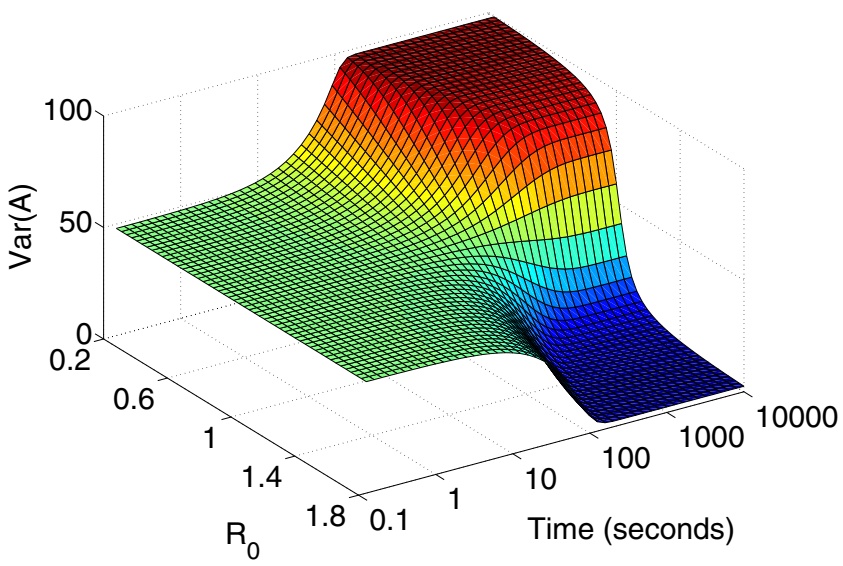

FIG. 4. (Color online) Mean value of $A$ in the push-pull system as a function of time and total enzyme level $\left(R_{0}\right)$. The GoldbeterKoshland point occurs at $R_{0}=1$. The switchlike behavior at steady state is clearly visible after the $t=1000$ point. Parameter values are $v_{r}=v_{b}=0.7, k_{+}=k_{-}=k_{+}^{\prime}=k_{-}^{\prime}=15, B_{0}=1, N=$ $100, A(0)=50$.

state for different levels of starting enzyme $R$. It is evident that the approach to steady state is slowed close to the ultrasensitive enzyme levels. Figure 5 shows the transient behavior of the variance of $A$ for the same parameter variation. Interestingly, we observe a sharp but transient increase in variance between 10 and $100 \mathrm{sec}$ for all values of $R_{0}$. However, as the system approaches steady state, only the systems near the ultrasensitive point have very high variance.

\section{CONCLUSION}

In summary, we have derived a general closed form expression for the solution of the 1D, one-step ME in terms of the eigenvalues of the matrix $A$. This suggests the problem is reduced to a symmetric tridiagonal eigenvalue problem rather than an eigenvalue and eigenvector problem. This allows us to solve the ME analytically in some cases, and otherwise it makes it possible to very quickly compute the solution. Indeed, in the dimerization example we studied, we solved the problem in between 1 and 3 orders of magnitude faster than the brute force method. The advantage is particularly apparent for systems with a large number of states.

We also looked at three examples from biochemical systems. In particular, we show that the ME for the push-pull system can be reduced to a $1 \mathrm{D}$, one-step ME with nonlinear

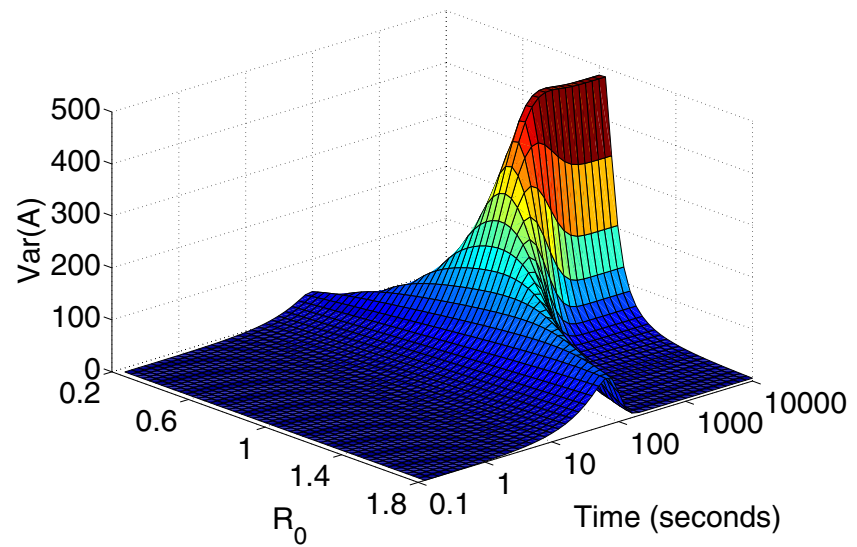

FIG. 5. (Color online) Variance of $A$ in the push-pull system as a function of time and total enzyme level $\left(R_{0}\right)$. The parameter values are the same as Fig. 4.

rate terms that can be solved efficiently with our method. We then explore transient behavior of this system in the zero-order ultrasensitive regime [27]. Interestingly, we observe a transient increase in variance of substrate, even far from the ultrasensitive values of enzymes. Previous, stochastic treatments of the push-pull system have focused mainly at the steady state $[26,28,29]$. Our method, is ideally suited for the study of the transient behavior. Although we derive our results using QSSA, our method is equally applicable to other approximations such as total QSSA [26].

If an analytical expression for the eigenvalues of an arbitrary tridiagonal matrix is obtained (currently this exists only for matrices with linear coefficients), it would remove the requirement for computation altogether. Since the calculation of these eigenvalues is the largest computational burden of the above method, this would speed up the process considerably. In addition, a general formula for the elements of the inverse of pentadiagonal, heptadiagonal, or other multidiagonal matrices would allow us to also solve the 1D ME for multistep systems, where the states go up or down by different numbers with each reaction.

Finally, a generalization of our approach to higher dimensions would greatly increase its applicability to more complex systems, where two or more chemical species interact with each other. The current work already hints at possible directions, but even two-dimensional systems require linear algebraic techniques significantly more complicated than those used above. We have made some progress in this direction, and this will be the subject of a subsequent paper.

[1] V. Shahrezaei and P. S. Swain, Curr. Opin. Biotechnol. 19, 369 (2008).

[2] D. J. Wilkinson, Nat. Rev. Genet. 10, 122 (2009).

[3] D. Gillespie, Physica A 188, 404 (1992).

[4] N. G. van Kampen, Stochastic Processes in Physics and Chemistry, 3rd ed. (Elsevier, Amsterdam, 2001).

[5] R. Grima, Phys. Rev. E. 84, 056109 (2011).

[6] C. W. Gardiner, Handbook of Stochastic Methods for Physics, Chemistry and the Natural Sciences (Springer, New York, 2004).

[7] R. Grima, P. Thomas, and A. V. Straube, J. Chem. Phys. 135, 084103 (2011).

[8] P. Whittle and J. R. Stat. Soc. Ser. B (Methodol.) 19, 268 (1957).

[9] D. A. McQuarrie, C. J. Jachimowski, and M. E. Russell, J. Chem. Phys. 40, 2914 (1964).

[10] R. Grima, J. Chem. Phys. 136, 154105 (2012).

[11] D. Schnoerr, J. Chem. Phys. 141, 024103 (2014).

[12] V. Shahrezaei and P. S. Swain, Proc. Natl. Acad. Sci. USA 105, 17256 (2008).

[13] P. Thomas, A. V. Straube, and R. Grima, BMC Sys. Biol. 6, 39 (2012).

[14] D. T. Gillespie, J. Comput. Phys. 22, 403 (1976). 
[15] B. Munsky and M. Khammash, J. Chem. Phys. 124, 044104 (2006).

[16] C. Moler and C. Van Loan, SIAM Rev. 20, 801 (1978).

[17] C. Moler and C. Van Loan, SIAM Rev. 45, 3 (2003).

[18] R. Basile, R. Grima, and N. Popovic, Bull. Math. Biol. 75, 1653 (2012).

[19] I. J. Laurenzi, J. Chem. Phys. 113, 3315 (2000).

[20] J. Murphy and M. O’Donohoe, IMA J. Appl. Math. 16, 57 (1975).

[21] F. W. Crawford and M. A. Suchard, J. Math. Biol. 65, 553 (2012).

[22] I. S. Dhillon, Ph.D. thesis, University of California, Berkeley, 1997.
[23] N. J. Higham, Functions of Matrices: Theory and Computation (SIAM, Philadelphia, 2008).

[24] R. A. Usmani, Linear Algebra Appl. 212, 413 (1994).

[25] A. H. El-Shaarawi, Appl. Environ. Microbiol. 41, 107 (1981).

[26] P. K. Jithinraj, U. Roy, and M. Gopalakrishnan, J. Theoret. Biol. 344, 1 (2014).

[27] A. Goldbeter and D. E. Koshland, Proc. Natl. Acad. Sci. USA 78, 6840 (1981).

[28] O. Berg, J. Paulsson, and M. Ehrenberg, Biophys. J. 79, 1228 (2000).

[29] T. Shibata and K. Fujimoto, Proc. Natl. Acad. Sci. USA 102, 331 (2005). 